\title{
Effective Image Compression Techniques using Pre- filtering of Daubechies Transform
}

\author{
Deepa N.R \\ PG Scholar, Dept of CSE \\ CIET, Coimbatore
}

\author{
Vasanthi Kumari.P \\ Assistant Professor, Dept of CSE \\ CIET, Coimbatore
}

\begin{abstract}
Image compression has become an active area of research in the field of Image processing particularly in the applications of medical and space sciences. Due to its importance, several image compression techniques were developed in the literature. But, majority of the conventional image compression approach available in the literature have poor visual quality and low PSNR value. In recent years, wavelet transform is observed to be very efficient in improving the performance of the image compression techniques. The main advantages of wavelet based methods lie in their energy compaction property and multi-resolution decomposition capability. The existing Haar transform based image compression approach has very low computational complexity but its compression performance is not significant. Improved SPIHT algorithm is used in this approach for the effective image compression of the image. This paper introduces a novel image compression approach using effective Daubechies Wavelet transform. For best performance in image compression, pre-filtering of wavelets transform is performed that combine a number of desirable properties, such as orthogonality and symmetry. The experimental results reveal that PSNR value and the visual quality of the proposed Improved SPIHT are very significant.
\end{abstract}

\section{Keywords}

Peak Signal to Noise Ratio, Daubechies Transform, Haar Transform, Set Partitioning in Hierarchical Tree.

\section{INTRODUCTION}

Analysis and compression of images is an important area of research in the field of image compression. Image analysis and data compression are developing fields which are extensively used in the applications such as pattern recognition, classification, statistical medical data analysis etc. [1]. The main aim of image compression is to diminish the redundancy of the image and to accumulate or broadcast data in a competent form. The main aim of image compression system is to minimize the storage quantity as much as possible, and the decoded image should be analogous to the original image.

Computer is becoming more and more important nowadays. Thus, the employment of digital images has become an important factor in the modern era. Moreover, storing and transferring the huge volume of image data has become a serious issue as the uncompressed multimedia data needs significant storage capacity and transmission bandwidth. Even though there is an extensive growth in mass storage density, the need for data storage capacity and data transmission bandwidth continues to go beyond the capabilities of the existing techniques. In addition, the extensive development of data intensive multimedia based web applications has forced the researchers to identify the method by means of the images in the web applications more efficiently. Internet teleconferencing, High Definition Television (HDTV), satellite communications and digital storage of movies are not feasible without a high degree of compression. These applications are far from realizing their full potential largely due to the drawbacks of traditional image compression techniques [2].

The image is a type of redundant data i.e. it consists of the same data from certain view point. Thus, through compression techniques, some of the redundant information contained in images can be eliminated. Image compression minimizes the size in bytes without degrading the quality of the image. The minimization in file size facilitates more images to be stored in a certain amount of memory space. It also minimizes the time essential for images to be sent over the Internet or downloaded from web pages.

Discrete Cosine Transform (DCT) in 1974 [3] has been an important achievement in the field of image compression. The DCT can be considered as a discrete time version of the Fourier Cosine series. It is a close relative of Discrete Fourier Transform (DFT) which is an approach for transferring a signal into elementary frequency components. Therefore, DCT can be calculated with a Fast Fourier Transform (FFT) like algorithm of complexity $O(n \log 2 n)$. Unlike DFT, DCT is real valued and offers a better approximation of a signal with fewer coefficients. There are several techniques in which image files can be compressed. There are two general compressed graphic image formats namely Joint Photographic Experts Group (JPEG) [4] and Graphic Interchange Format (GIF) for the use in the Internet.

More recently, the wavelet transform has considered as an important technique within the field of image analysis. Wavelets are a mathematical tool for hierarchically decomposing functions. Wavelet Image Processing facilitates computers to accumulate an image in many scales of resolutions, thus decomposing an image into various levels 
and types of details and approximation with different valued resolutions. Wavelets facilitate image compression using less storage space with more details of the image.

Wavelets have recently been applied to several problems in Computer Graphics including image editing and compression, surface reconstruction, global illumination, and animation [5]. Wavelet-based coding [6] offers considerable enhancements in picture quality at higher compression ratios. Recently, a wide variety of powerful and sophisticated wavelet-based approaches for image compression have been developed and implemented.

This paper uses a wavelet based approach for effective image compression due to the following advantages of the wavelets.

- Wavelet coding approaches at higher compression avoid blocking artifacts.

- Wavelet coding are better matched to the Human Visual System (HVS) characteristics.

- Wavelet based compression facilitate parametric gain control for image softening and sharpening.

- Wavelet-based coding is more robust under transmission and decoding errors, and also allows progressive transmission of images.

- Wavelet compression is very competent at low bit rates.

- Wavelets offer an efficient decomposition of signals earlier to compression.

SPIHT algorithm is used in this research work for better efficiency. SPIHT algorithm produces a pyramid structure based on a wavelet decomposition of an image. It has been discussed that the wavelet coefficients at the top of the pyramid have a strong spatial relationship with their children. This approach becomes very significant by iteratively searching for significant pixels throughout the pyramid tree. In order to improve the performance of the system, Improved SPIHT algorithm is used. This paper introduces a novel Improved SPIHT image compression technique using effective Daubechies Wavelet transform.

\section{LITERATURE SURVEY}

A reformulation of the Haar transform is exploited to propose systolic arrays to carry out the process of data compression by Vergara Villegas et al., [7]. Initially a triangular array is developed for the normalized 1-D transform and subsequently it is then extended to generate an inverse transformation. Area-efficient and unrolled array designs utilizing the 1-D arrays are exploited to build up high-throughput, areaefficient arrays with $100 \%$, effectiveness which generate one transformed data item per clock cycle. The 1-D designs are then integrated into a 2-D design for image compression with the use of row and column operations. At last the arrays are increased with a simple thresholding design which generates a compressed lossy output. High throughput can be accomplished. A comprehensive compression/decompression of the data can be carried out using the arrays in combination with a thresholding array which adds $O\left(\mathrm{~m}^{2}\right)$ cells and an additional latency of $2 \mathrm{~m}$ steps. The design is scalable in that $\mathrm{m}$ can be selected to suit manufacturing capabilities.

De Queiroz et al., [8] presented the model and the accomplishment of an object classification system that with the dissimilarity to the traditional systems uses images obtained from a lossy compress/decompress process. The domain transformation is prepared with Daubechies D4 wavelet transform and the coefficients coding is done using the Embedded Zerotree Wavelet (EZW) approach. This model recommends as main advantages a saving of until $50 \%$ of the image entire storage space and even with the information loss the images are typically very comparable to the originals. The system facilitates obtaining extremely near classification results to those results acquired using the original images. With the purpose of testing the model, the problem of apple classification is considered. Apples are categorized into two categories: bad and good quality based on specified criteria which are evaluated with a process of extraction and selection of features and the implementation of a voting algorithm.

The implementation of Wavelet-based still image compression is turning out to be ideal since the wavelet theory research continues to increase in the last decade. According to the analysis of the fundamental theory of wavelet transform, the application of wavelet transform in still image compression, and executed the Daubechies 9/7 wavelet transform of three decomposition by means of $\mathrm{VC}++$ platform programming by Lin and Smith [9].

\section{METHODOLOGY}

A rotation structure for prefiltering the Daubechies transforms which both enhances frequency selectivity and eliminates block distortions. This rotational transform can be considered as a pre-filter extension of the 2-point DCT that uses the simplicity of rotation operations, resulting in an 8-tap Periodically Time-Varying (PTV) filter bank [10], [11] with attractive properties. The proposed transform is basically multiplier free and symmetric extension can be carried out easily. The performance of the wavelet filter is higher than the $5 / 3$ filters with comparable complexity. In comparison to the 9/7 filters, its complexity is notably lower and the performance is almost as good when employed in JPEG2000 coders.

The principle behind transform image coding is based on two factors: 1) The correlation among the transform coefficients is reduced so that redundant information does not have to be coded repeatedly; and 2) Due to the energy compaction property, it is possible to code only a fraction of the transformed coefficients without producing serious distortion.

The main problem in D4 is handling the edges; to solve this, we treat the data set as if it is mirrored at the ends. The main components in the proposed approach are prefiltering, compression, Decompression and Reverse prefiltering.

\subsection{Prefiltering}

Many techniques have been developed to improve coding efficiency and to avoid or reduce blocking effects. Most can be classified into two distinct categories: i) using a global transform (more accurately, transforms with overlapping basis functions) and ii) using pre- and post-processing techniques. Algorithms in the first approach improve reconstruction quality by employing either the wavelet transform or the 
lapped transform (LT) in signal decomposition and reconstruction.

- Pre- and post-filter are constructed in modular cascaded stages, leading to minimal hardware/software modifications and simple future upgrades.

- $\quad$ Pre- and post-processing retain all flexible features of block-based approaches and add on top a high level of adaptivity in signal decomposition.

- Blocking artifacts are eliminated with post-filtering along the block boundaries, while ringing artifacts can be controlled by varying the number of borrowing samples.

This rotational transform can be viewed as a prefilter extension of the 2-point DCT that exploits the simplicity of rotation operations.

The prefiltering is performed by plane rotations so that the resulting transform is an overlapped orthogonal transform. In order to keep complexity low, only one rotation angle is employed. Specifically, odd samples are consecutively rotated twice by $+\theta$ and even samples are consecutively rotated twice by $-\theta$. Thus, in this approach, the pre-filtered image is obtained. This is given as input to the next module namely Compression.

\subsection{Compression}

In this project, the Daubechies D4 wavelet transform is used in order to change the domain representation of an image.

The Daubechies D4 wavelet transform has four wavelet and scaling function coefficients. The scaling function coefficients are show in equation 1 , and the wavelet function coefficients in equation 2 .

$$
h_{0}=\frac{1+\sqrt{3}}{4 \sqrt{2}}, \quad h_{1}=\frac{3+\sqrt{3}}{4 \sqrt{2}}, \quad h_{2}=\frac{3-\sqrt{3}}{4 \sqrt{2}}, \quad h_{3}=\frac{1-\sqrt{3}}{4 \sqrt{2}}
$$

(1)

$$
\mathrm{g}_{0}=\mathrm{h}_{3}, \quad \mathrm{~g}_{1}=-\mathrm{h}_{2}, \quad \mathrm{~g}_{2}=\mathrm{h}_{1}, \quad \mathrm{~g}_{3}=-\mathrm{h}_{0}
$$

An image is taken and divided it in $8 \times 8$ sub block in order to obtain a matrix as shown in figure 1 below, then apply Daubechies D4 first in rows and then in columns.

\begin{tabular}{|rrrrrrrr|}
\hline 64 & 2 & 3 & 61 & 60 & 6 & 7 & 57 \\
9 & 55 & 54 & 12 & 13 & 51 & 50 & 16 \\
17 & 47 & 46 & 20 & 21 & 43 & 42 & 24 \\
40 & 26 & 27 & 37 & 36 & 30 & 31 & 33 \\
32 & 34 & 35 & 29 & 28 & 38 & 39 & 25 \\
41 & 23 & 22 & 44 & 45 & 19 & 18 & 48 \\
49 & 15 & 14 & 52 & 53 & 11 & 10 & 56 \\
8 & 58 & 59 & 5 & 4 & 62 & 63 & 1 \\
\hline
\end{tabular}

\section{Figure 1: Matrix used for D4 example}

Take the first row of the matrix $(64,2,3,61,60,6,7,57)$, then obtain the D4 transform. In the first pass of D4 a scaling function is applied to the complete row and the first part of the array is changed.

The result is shown in figure 2a. After this, apply the wavelet function. The results are shown in figure $2 b$. Then, repeat the algorithm with the first half of the array (applying scaling figure $2 \mathrm{c}$ and wavelet function figure $2 \mathrm{~d}$ ) in order to obtain the final transformation.

\begin{tabular}{|c|c|c|c|c|c|c|c|}
\hline \multicolumn{9}{|c|}{ a) } \\
\hline $\mathbf{0}$ & $\mathbf{1}$ & $\mathbf{2}$ & $\mathbf{3}$ & $\mathbf{4}$ & $\mathbf{5}$ & $\mathbf{6}$ & $\mathbf{7}$ \\
\hline 25.361008 & 65.148498 & 28.189536 & 65.148558 & -35.681596 & 33.232196 & -30.782706 & 38.889050 \\
\hline 64.633974 & 65.366025 & -25.767949 & -28.5 & & & & \\
\hline
\end{tabular}

Figure 2: Daubechies D4 forward transform results.

\subsection{SPIHT}

Then the image is compressed using the SPIHT algorithm. SPIHT is the wavelet based image compression technique. It offers the Highest Image Quality, Progressive image transmission, fully embedded coded file, Simple quantization algorithm, fast coding/decoding, completely adaptive, Lossless compression, exact bit rate coding and Error protection $[12,13]$.

SPIHT utilizes three lists namely the List of Significant Pixels (LSP), List of Insignificant Pixels (LIP) and List of Insignificant Sets (LIS). These are coefficient location lists that comprises of their coordinates. After the initialization, the algorithm consists of two stages for each level of threshold namely the sorting pass (where lists are prearranged) and the refinement pass (which does the actual progressive coding transmission). The result is in the form of a bit stream. It is able to recover the image completely by coding all bits of the transform. But, the Haar wavelet transform provides ideal reconstruction only if its numbers are stored as infinite imprecision numbers.

The flowchart of SPIHT is presented in Figure 3. 
Threshold $T_{n}=T_{n-1} / 2$

Reconstructive Value: $R_{n}=R_{n-1} / 2$

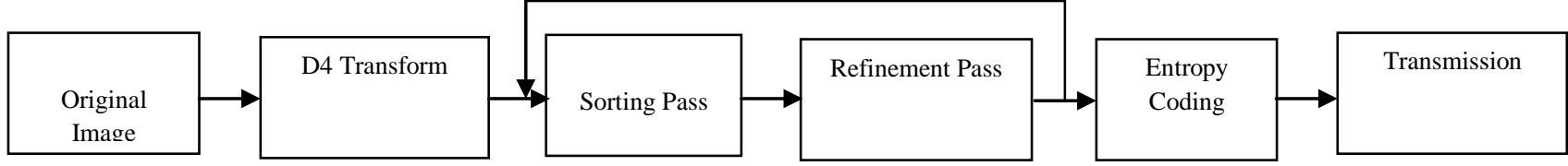

Figure 3: SPIHT Algorithm

First step, the original image is decomposed into ten sub-bands. Then, the method finds the maximum and the iteration number. Second step, the method puts the DWT coefficients into sorting pass that finds the significance coefficients in all coefficients and encodes the sign of these significance coefficients. Third step, the significance coefficients that be found in sorting pass are put into the refinement pass that use two bits to exact the reconstruct value for closing to real value. The front second and third steps are iterative, next iteration decreases the threshold $\mathrm{Tn}=\mathrm{Tn}-1 / 2$ and $\mathrm{Rn}=\mathrm{Rsn}-1 / 2$ the reconstructive value Forth step, the encoding bits access entropy coding and then transmission. Thus, the image is compressed in this process.

\section{Limitations of SPIHT Coding Scheme}

1. When scanning the list of insignificant pixels (LIP), list of insignificant sets (LIS), or list of significant pixels (LSP), the repeated coefficient comparison can increase complexity of the algorithm.

2. The coefficients put into LIP at last scanning process which are smaller than the present threshold will result in redundancy.

3. Early coding for non-important coefficients in SPIHT will influence the performance of channel coding, particularly unequal error protection (UEP) [14].

\section{Improved SPIHT Coding Scheme}

In this image compression technique, improved SPIHT technique is used.

In Normal SPIHT - LIP comprises of approximation coefficients with nth level detail coefficients. However, LIS will have nth level detail coefficients. In Improved SPIHT- LIP comprises of $1^{\text {st }}$ and $2^{\text {nd }}$ row which is again approximation coefficients with nth level detail coefficients. However, LIS will have $2^{\text {nd }}$ row which is nth level detail coefficients. The improved SPIHT technique has the following alterations. SPIHT codes four coefficients and then shifts to the next four ones. Thus, observes the four coefficients as a block. The maximum of them is considered as the compared threshold will minimize number of comparison, which is related with the distribution of coefficient matrix. Especially, when the maximum in the block is lesser or equal to the present threshold, the block will be coded with only one bit rather than four zeros.

$\mathrm{LlB}=\{\mathrm{B}(\mathrm{O}, \mathrm{O}), \mathrm{B}(0,2), \mathrm{B}(2,0), \mathrm{B}(2,2)\}, \mathrm{LlS}=\{\mathrm{D}(0,2), \mathrm{D}(2,0)$, $\mathrm{D}(2,2)\}, \mathrm{T}=2 \mathrm{n}, \mathrm{Cij}$ is wavelet matrix coefficient and LSP is empty. $\mathrm{n}$ is expressed in (1).

$$
n=\left|\log _{2}\left(\max _{(i, j)}\left|C_{i, j}\right|\right)\right|
$$

$\left|C_{i, j}\right|$ is the matrix coefficient after DWT and $(\mathrm{i}, \mathrm{j})$ is the coordinate of $C_{i, j}$.

Sorting pass: The sorting pass comprises of two tests: the LIB test (LIBT) and LIS test (LIST). The LIBT will code the block or coefficients in blocks, while the LIST mainly disposes the sets in LIS.

Thus, this proposed approach can minimize redundancy to a significant extend. When calculating the maximum threshold, the improved algorithm can start the maximum of every block. Therefore, it can clearly minimize number of comparison when scanning and coding zero trees.

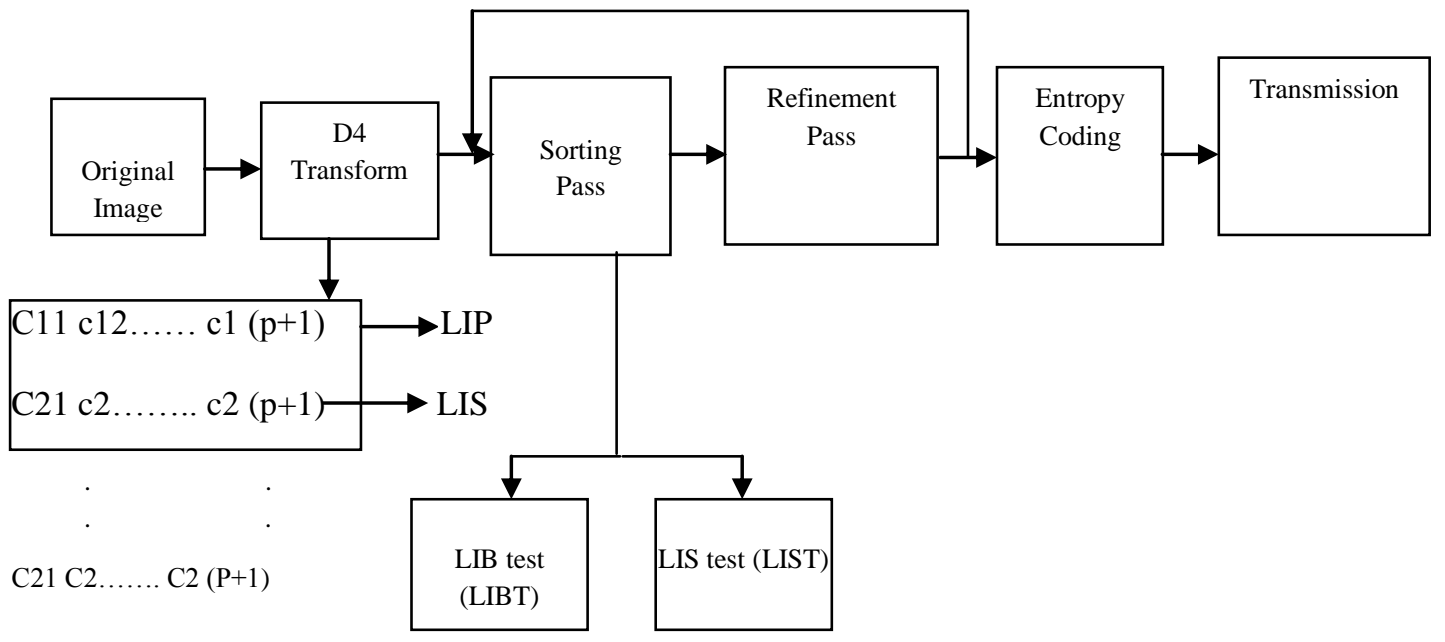

Figure 4: Improved SPIHT 


\subsection{Decompression}

This process is the reverse to the compression technique. After SPIHT, it is necessary to transform data to the original domain (spatial domain), to do this the inverse Daubechies D4 wavelet transform is applied first in columns and secondly in rows.

\subsection{Reverse Pre-Filtering}

This procedure is entirely reverse to the Pre-Filtering Process. The inverse transform is obtained by reversing the order of operations and inverting the signs of the rotation angle. Thus, the inverse transform has exactly the same computational complexity as the original transform.

Thus the original image is obtained through effective image compression technique.

\section{EXPERIMENTAL RESULTS}

The experiment is carried out in MATLAB 7.0. The performance of the proposed approach is evaluated based on the PSNR value. The proposed approach is compared with the image compression using Haar transform. Standard images like Lena, Mandril and Woman of size $256 \times 256$ are taken for experimentation.

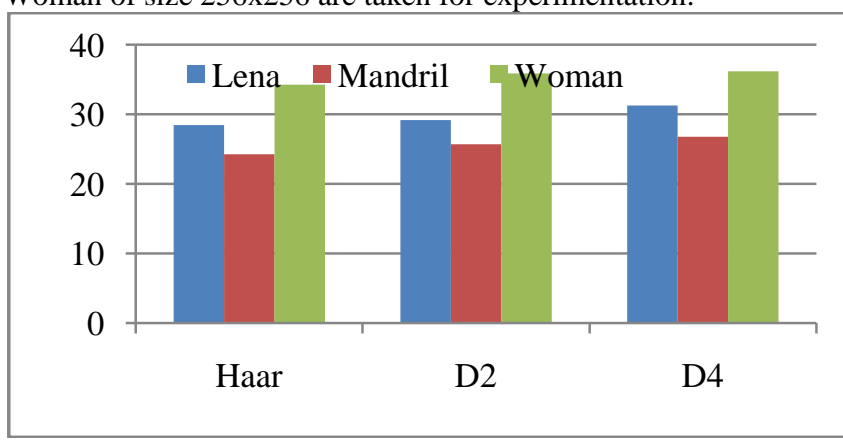

Figure 5: PSNR Evaluation of the Image Compression Techniques

Figure 5 shows the PSNR value comparison of the proposed Daubechies and the existing Haar approach. It is observed that the proposed approach provides better PSNR value when compared with the existing technique.

The MSE value comparison is shown in figure 6. It is observed from the figure that the MSE value of the proposed Daubechies D4 approach is very less when compared with the existing Haar Transformation technique and Daubechies D2 transformation.

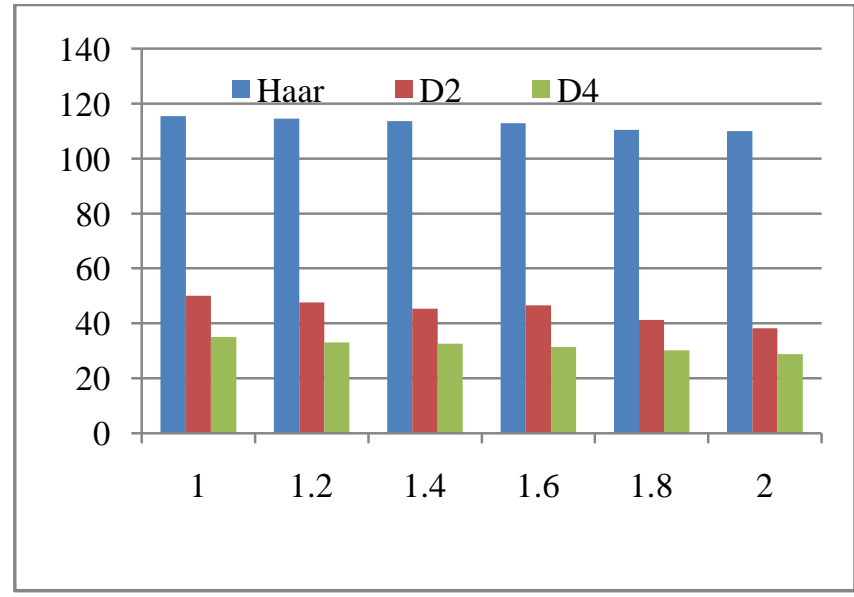

Figure 6: MSR Evaluation of the Image Compression Techniques for Lena Image
Evaluation results using SPIHT and Improved SPIHT with Haar, D2 and D4 transformation.

The PSNR value using the SPIHT and Improved SPIHT algorithms is shown in figure 7 and Table 1.

\section{Table 1: PSNR Value Evaluation}

\begin{tabular}{|c|c|c|c|}
\hline \multirow{2}{*}{ Image } & \multicolumn{3}{|c|}{ PSNR (dB) } \\
\cline { 2 - 4 } & $\begin{array}{c}\text { Haar } \\
\text { Transform } \\
\text { with } \\
\text { SPIHT }\end{array}$ & $\begin{array}{c}\text { D2 } \\
\text { Transform } \\
\text { Modified } \\
\text { SPIHT }\end{array}$ & $\begin{array}{c}\text { D4 } \\
\text { Transform } \\
\text { with } \\
\text { Modified } \\
\text { SPIHT }\end{array}$ \\
\hline $\begin{array}{c}\text { Lena } \\
(256 * 256)\end{array}$ & Lena & Mandril & Women \\
\hline $\begin{array}{c}\text { Mandril } \\
(256 * 256)\end{array}$ & & & \\
\hline Woman & & & \\
$(256 * 256)$ & & & \\
\hline
\end{tabular}

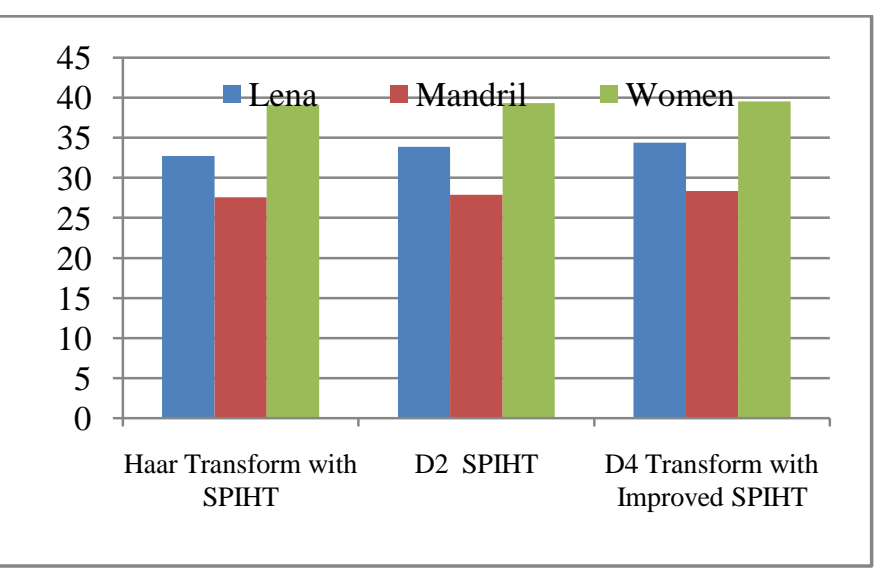

Figure 7: PSNR Value comparison of SPIHT and Improved SPIHT

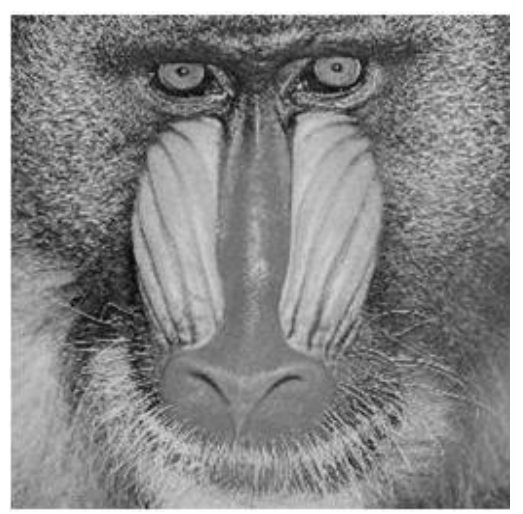

Figure9: (a) Original Mandril Image 


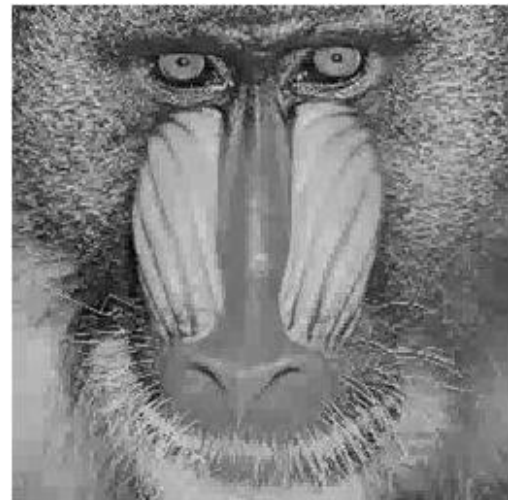

Figure 9: (b) Reconstructed Mandril Image using Haar with SPIHT algorithm

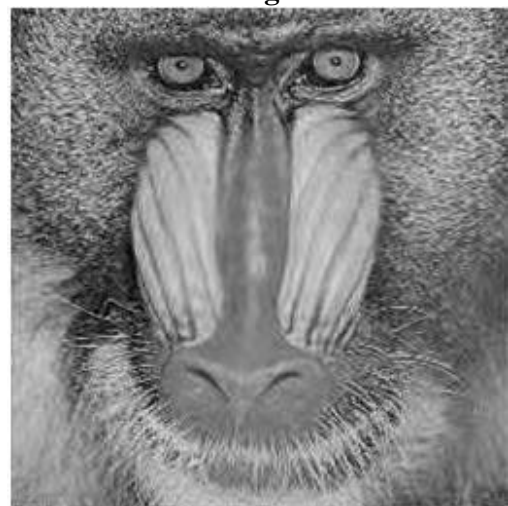

Figure 9: (c) Reconstructed Mandril Image using Daubechies D4 with Improved SPIHT algorithm

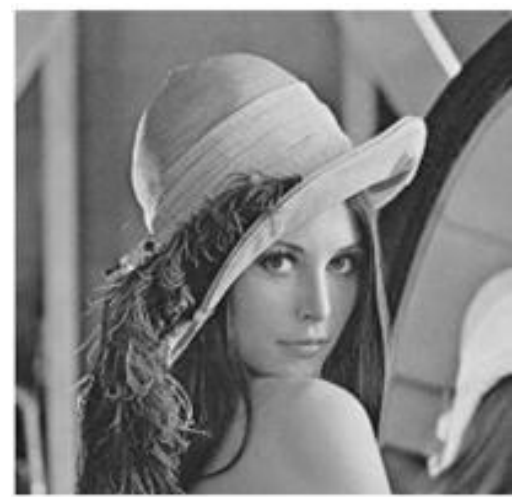

Figure 10: (a) Original Barbara Image

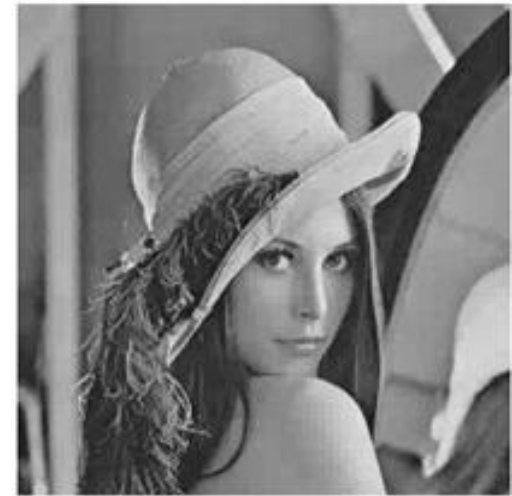

Figure 10: (b) Reconstructed Lena Image using Haar with SPIHT algorithm

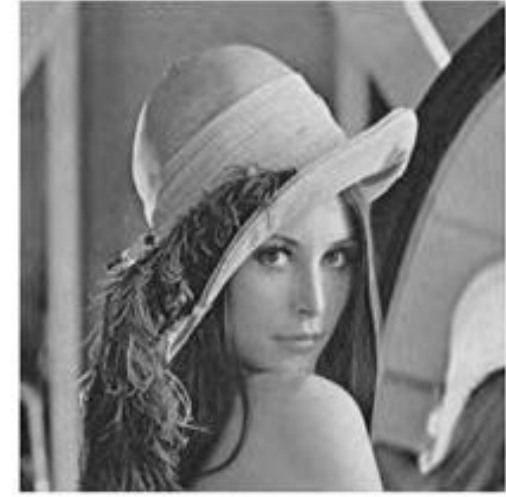

Figure 10: (c) Reconstructed Lena Image using Daubechies D4 with Improved SPIHT algorithm

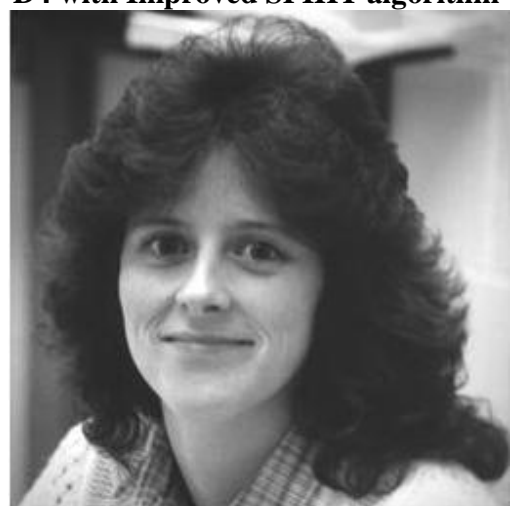

Figure 11: (a) Original Woman Image

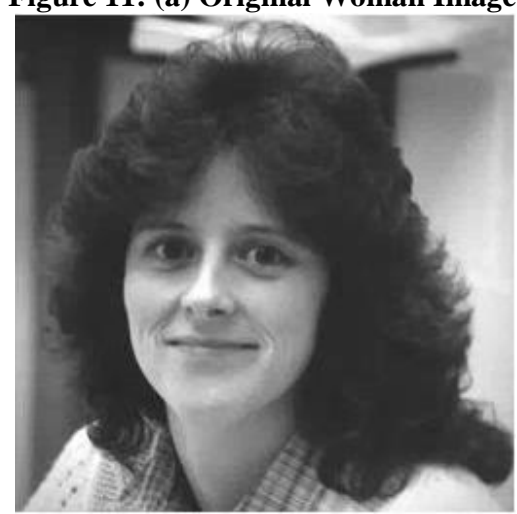

Figure 11: (b) Reconstructed Woman Image using Haar with SPIHT algorithm

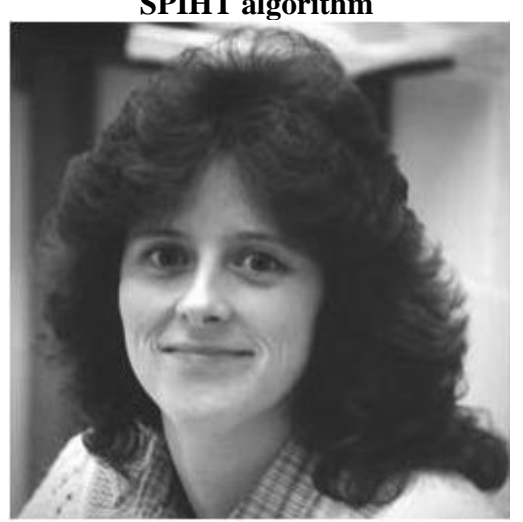

Figure 11: (b) Reconstructed Woman Image using Daubechies D4 with Improved SPIHT algorithm 


\section{CONCLUSION}

A novel image compression technique is introduced in this approach for providing better security and visual quality. Prefiltering technique is used in this technique for providing better efficiency and significance. Improved SPIHT algorithm is used for compression. The performance of the proposed approach is evaluated with standard images like Lena and Pepper. It is observed from the result that the proposed Daubechies approach provides high PSNR values. Moreover, MSE value of the proposed approach is also very less when compared with the existing technique. The results are then evaluated for SPIHT and Improved SPIHT algorithms. Moreover, Daubechies D2 and D4 transformations are also compared in this approach. It is observed that the proposed Daubechies transform with Improved SPIHT algorithm provides better results in terms of PSNR value.

\section{REFERENCES}

[1] S Nanavati and P Panigrahi, Wavelets: Applications to image compressionI, Resonance, Vol.10, No.2, pp.52-61, 2005

[2] Debin Zhao; Wen Gao; Chan, Y.K.; "Morphological representation of DCT coefficients for image compression", IEEE Transactions on Circuits and Systems for Video Technology, Volume: 12, Issue: 9, 2002.

[3] Wen-Chien Yen; Shen-Chuan Tai; "DCT-based image compression using wavelet-based algorithm with efficient deblocking filter", Fourth Annual ACIS International Conference on Computer and Information Science, 2005.

[4] Minasyan, S.; Astola, J.; Guevorkian, D.; "An image compression scheme based on parametric Haar-like transform", IEEE International Symposium on Circuits and Systems, 2005. ISCAS 2005.

[5] R. L. de Queiroz, T. Q. Nguyen, and K. R. Rao, "The GenLOT: Generalized linear-phase lapped orthogonal transform," IEEE Trans. Signal Process., vol. 44, no. 3, pp. 497-507, Mar. 1996.

[6] J. M. Shapiro, "Embedded image coding using zerotrees of wavelet coefficients," IEEE Trans. Signal Processing, vol. 41, pp. 3445-3462, Dec. 1993

[7] Vergara Villegas, O.O.; Pinto Elias, R.; Cruz Sanchez, V.G.; "Apple Classification System with EZW and Daubechies D4 Lossy Image Compression”,. 16th
International Conference on Electronics, Communications and Computers, 2006. CONIELECOMP 2006.

[8] R. L. de Queiroz, T. Q. Nguyen, and K. R. Rao, "The GenLOT: Generalized linear-phase lapped orthogonal transform,” IEEE Trans. Signal Process., vol. 44, no. 3, pp. 497-507, Mar. 1996.

[9] J. J. Lin and M. J. T. Smith, "New perspectives and improvements on the symmetric extension filter bank for subband/wavelet image compression," IEEE Trans. Image Process., vol. 17, no. 2, pp. 177-189, Feb. 2008.

[10] K. Nayebi, T. P. Barnwell, and M. J. T. Smith, "Analysis-synthesis systems with time-varying filter bank structures," in Proc. Int. Conf. Acoust., Speech Signal Process., Mar. 1992, vol. 4, pp. 617-620.

[11] I. Sodagar, "Analysis and design of time-varying filter banks," Ph.D. dissertation, Georgia Inst. Tech., Atlanta, Nov. 1994, pp. 21-24.

[12] Sandeep Kumar, "Image Compression Based on Improved Spiht and Region of Interest", A thesis, 2011.

[13] H. L. Xu, S. H. Zhong, "Image Compression Algorithm of SPIHT Based on Block-Tree," Journal of Hunan Institute of Engineering, vol. 19(1), pp.58-61, 2009.

[14] Hualiang Zhu, Chundi Xiu and Dongkai Yang, "An improved SPIHT algorithm based on wavelet coefficient blocks for image coding", International Conference on Computer Application and System Modeling (ICCASM), 2010.

\section{AUTHORS PROFILE}

Deepa N.R is currently pursuing M.E, Computer Science and Engineering in Coimbatore Institute of Engineering and Technology (C.I.E.T), Coimbatore. She received her B.E degree in Computer Science and Engineering at Coimbatore Institute of Engineering and Technology, Coimbatore. Her Research areas are Image Processing and Data Mining.

Vasanthi Kumari $\mathbf{P}$ is working as an Assistant Professor in Coimbatore Institute of Engineering and Technology (C.I.E.T), Coimbatore. She received her M.E degree in Computer Science and Engineering at Anna University, Chennai in the year of 2007, MCA and B. Sc. (Physics) degree from Bharathiar University, Coimbatore in the year of 1999 and 1996. She has 12 years of teaching experience. Her research areas are image processing, data structures and data mining and warehousing. 